\title{
CONCEALMENT IN CONSULTATIVE ENCOUNTERS IN NIGERIAN HOSPITALS ${ }^{1}$
}

\author{
Akin Odebunmi ${ }^{2}$
}

\begin{abstract}
Although communication in medical practice is reputed for exactitude and objectivity, many doctors in several countries make equivocal, concealing utterances in certain situations when relating with clients. This phenomenon, despite its importance in doctor-client interaction, has received little attention from language scholars who have discussed concealment mainly as a strategy in news delivery. The present study examines concealment items in the interaction between doctors and clients in South-western Nigerian hospitals and their pragmatic implications for medical communication in Nigeria. Fifty (50) conversations between doctors and clients on several ailments were tape-recorded in the six states of South-western Nigeria. Structured and unstructured interviews were conducted with selected doctors and clients. The corpus was examined for the linguistic and pragmatic resources deployed by doctors in concealing information, and was analysed using Jacob Mey's theory of pragmeme and insights from the literature on news delivery strategies. Concealment was found to take place between doctors and clients in a two-phase mode: Referential and pragmatic. Utterances which have descriptive forms at the referential level assume subjective and divergent shades in the context of concealment at the pragmatic level. Nine concealment strategies (jargonisation, veiling, forecasting, mitigation, stalling, normalisation, dysphemisation, euphemisation and doublespeak) were found to be employed to achieve four broad goals: Preventive, palliative, culture-compliant and confidential with respect to 25 diseases /medical procedures. Concealment in consultative encounters takes into account the socio-psychological security needs of clients and attends positively to clients' cultural expectations.
\end{abstract}

Keywords: Concealment; Doctor-client interaction; Pragmeme; Strategies; Socio-psychological security.

\section{Introduction}

Business in medical practice is a two-way affair: Scientific and social. In its scientific angle, medicine deals with diagnoses and treatment of diseases, while in its social slant, it builds a relationship between the doctor and his/her clients through communication. Much of this social communication relates to the delivery of good and bad news in the hospital.

\footnotetext{
${ }^{1}$ This paper came out of the research I conducted on "The Pragmatics of Hospital Interactions in Nigeria" at the Freiburg Institute for Advanced Studies (FRIAS), University of Freiburg, Germany between April 2010 and March 2011 with the Alexander von Humboldt Georg Forster Fellowship award.

${ }^{2}$ I am grateful to Prof. Peter Auer, my host, for excellent advice. I also appreciate the useful comments I received from colleagues at the University of Bayreuth, Germany and FRIAS where I presented versions of this paper in October, 2010 and February, 2011 respectively.
} 
A variety of strategies have been developed to present news in the hospital (Maynard 1991, 2005, etc). These strategies are rooted in medical ethics, which states that a patient be given information about his/her condition, in almost all cases, in plain language. Despite this instruction, doctors in several parts of the world conceal information from patients through non-delivery, stalling (Maynard 1991, 1996) or veiling (Odebunmi 2008). Concealment, in the context of the present research, refers to the doctor-controlled act of withholding information from or providing unclear information to patients or their relations

In a way, there is little justification for news concealment by doctors. The World Medical Association's (WMA's) stance on concealment is that news can be withheld from patients, in certain cases, if it is established that they will harm themselves or commit suicide (Williams 2009). This presents some good ground for concealment to enable doctors protect their patients. But doctors, many times, do not limit themselves to this radius. In certain places, for example Nigeria, news is witheld in many more instances than stated by the WMA. Stalling or veiling the news is decided by the doctor as medical ethics itself leaves much ethical behaviour open to discretional judgement.

The practice of concealing news is very frequent among many Nigerian doctors, but it is not exclusive to them. In almost all countries, one form of concealment or another is practised. It is, however, more pronounced in some than others and practised more with respect to some conditions than others. According to Ong et.al. (1995: 906), "oncologists deliberately withhold information from their patients on the assumption that total disclosure will cause strong negative reactions on the side of the patients". Maynard (1996) notes that in some countries, patients elect to have bad news concealed. Thus, doctors, as a response to this preference, withhold information in some instances to "protect outsiders (patients and their families) from potentially detrimental knowledge" (Maynard 1996: 124). This takes several forms depending on the practice in each country. Maynard (1996) reports the situations in Ethiopia, Japan, and Italy. In Ethiopia, what is avoided is the sudden disclosure of the cancer bad news, not the news itself. Information is thus withheld until the right time, and disclosure strategies are decided upon among family members who must have been told earlier by the doctor. In both Japan and Italy, physicians sometimes do not announce cancer diagnoses because they assume patients already know and only avoid being told.

According to Maynard (1996: 125), "cross-cultural accounts suggest that the main reason for not telling patients their cancer diagnoses may be to prevent depression and preserve hope". He further notes that in some countries, news is not withheld completely "but that the delivery is properly staged, the quality of social relationships in which the informing occurs is maintained, and the realization occurs through other modes than verbal and cognitively logical ones" (Maynard 1996: 125; Good et al. 1990).

In the Nigerian hospital, doctors differ in their approach to bad news delivery. Some announce the news bluntly; some conceal it through various linguistic camouflages. While some studies, for example, Odebunmi (2003, 2005 and 2006b) have examined aspects of the linguistic means employed to camouflage the truth, no study has been exclusively devoted to concealment in consultative encounters. The relative neglect applies to the literature on medical discourse in general. The object of the present paper is, therefore, to examine the pragmatic strategies employed by doctors in South-western Nigeria to achieve concealment goals. Studying these strategies in the 
Nigerian hospital will not only reveal pragmatic peculiarities of the Nigerian hospital but will also show how Nigerian doctors manage bad news vis-à-vis their relationships with their clients and prescriptions of medical ethics.

\section{Methodology and design}

Fifty conversations between doctors and patients on several ailments were tape-recorded in the six states of South-western Nigeria: Oyo, Osun, Lagos, Ogun, Ondo and Ekiti in 2002 and 2009. After the recordings, about 30 of these doctors granted oral (unstructured) interviews on the use of language in the hospital with particular emphasis on how and why information was concealed from patients. Also covered in the interviews were questions on words, expressions and other methods used in concealing information. Structured interviews were conducted with about 150 doctors randomly selected in the hospitals. Unstructured interviews and structured interviews were granted by five and 100 patients respectively. Designed to confirm some of the information supplied by doctors, these interviews specifically asked for English and non-English expressions doctors used, together with other methods they employed, to conceal information from patients. The data were compiled into a corpus of about 300,000 words, and were examined for the linguistic and pragmatic resources deployed by doctors in concealing information. The analysis was based on Jacob Mey's (2001) theory of pragmeme and insights from the literature on news delivery strategies in hospitals.

In the next section (i.e. 3), I devote my attention to a review of research on news concealment in the hospital. In section 4, I provide the theoretical framework for the research. In Section 5, I analyse the data, and in section 6, I conclude the research by pointing out the pragmatic implications of concealment discourse for medical communication in Nigeria. Some aspects of Gricean pragmatics, relevance theory and Leechian politeness principles were found useful at certain points of the analysis.

\section{Studies on news delivery and concealment in hospital interaction}

News delivery is a doctor-based act performed when the health status of a client has been established. It is an announcement to the client of the diagnosis and sometimes the prognosis of their condition. There is a robust literature on news delivery in general, but there is an extremely scanty one on concealment in news delivery.

Attention to bad (and good) news delivery strategies has been paid by scholars such as Frankel (2001); Heath (1992); Heritage and Stivers (1999); Leppanen (1998); Maynard (1989, 1991, 1996, 2003, 2005,); Perakyla (1998); Stivers (1998); Heritage and Maynard (2006); Odebunmi (2008). Some of the interactional formats identified in the literature with respect to bad news delivery include generic News Delivery Sequence, Perspective Display Sequence, forecasting, bluntness and stalling. A generic News Delivery Sequence (NDS) is acquired, by doctors, overtime, without pedagogy, through "... participation in society and becoming competent at conversations" (Maynard 2005: 250). It consists of four talk segments: announcement, announcement response, elaboration and assessment. Both the doctor and the patient are involved in the process. The Perspective Display Sequence (PDS) involves the doctor inviting the 
view of a client about their condition, which enables him/her to assess the client's perspective enroute to announcing the diagnosis (cf. Maynard 1991).

Forecasting provides clues about the diagnosis or news, thereby "warning that bad news is forthcoming without keeping the recipient in a state of indefinite suspense" (Maynard 1996: 109). Bluntness involves announcing the news baldly without taking the client's perspectives and emotions into consideration. According to Maynard (1996: 119), this may raise apprehensions which include "taking the news as a joke, blaming the messenger or maintaining ordinariness". Stalling involves withholding the news thereby keeping the client in a state of suspense. It is capable of causing such misapprehensions as normalisation and self-blame (Maynard 1996). With respect to normalisation, the client makes incorrect, sometimes positive, inferences especially if euphemisms are used. Self-blame occurs when clients have no information about their own or their relation's conditions from doctors and resort to blaming themselves for the problem.

While some of these strategies have been linked to concealment, there has hardly been any research exclusively devoted to concealment in the hospital set-up. Maynard (1991) identifies two aspects of stalling as part of his Perspective Display Sequence project, what I have called "non-informing" and "euphemistic informing", but he does not develop them fully as they are integrated with larger news delivery strategies. Odebunmi (2003), working on the broad medical practitioners' (doctors and nurses) communicative behaviours towards clients in Nigerian hospitals, identifies veiling, through euphemisms and vagueness, as a method of concealing bad news in the hospital. In more specific studies on diagnostic news delivery (Odebunmi 2006b and Odebunmi 2008, respectively), he only identifies double-speak, and veiling and hedging as concealment strategies. For example, Odebunmi (2006b) identifies two kinds of lexical items doctors use in Nigerian hospitals: Words/expressions are used with the intention that patients/clients would understand and/or are used with the intention that clients would not understand. In the first case, doctors use plain words which are synonyms of the strictly technical words in medicine and, sometimes, in addition, provide clear explanations on subjects of interaction. This happens mostly when mild ailments and non-life threatening diseases are at issue. Sometimes however, plain communication occurs with respect to terminal, life threatening ailments. The choice of communication mode often strictly depends on the affordances (Mey 2001) of the context of interaction. The second type of word choice involves doctors speaking in a non-plain way, using medical jargon and other technical lexical items in communicating with clients to make comprehension impossible. This relates directly to the issue of concealment of information.

\section{Theoretical anchor: The pragmatic act theory}

The pragmatic act theory is a theory of context which considers the verbal behaviour of an individual within the affordances of the context. In Mey's (2001: 43) words, "the context determines what one can say and what one cannot say". Taking the theory beyond the limitations of the speech act theory (cf. Fairclough 1989; Odebunmi 2008), Mey conceives of context as action: 
Context is more than just reference. Context is action. Context is about understanding what things are for; it is also what gives our utterances their true pragmatic meaning and allows them to be counted as true pragmatic acts (Mey 2001: 41).

There are two parts to Mey's (2001) theory of a pragmeme: Activity and textual.
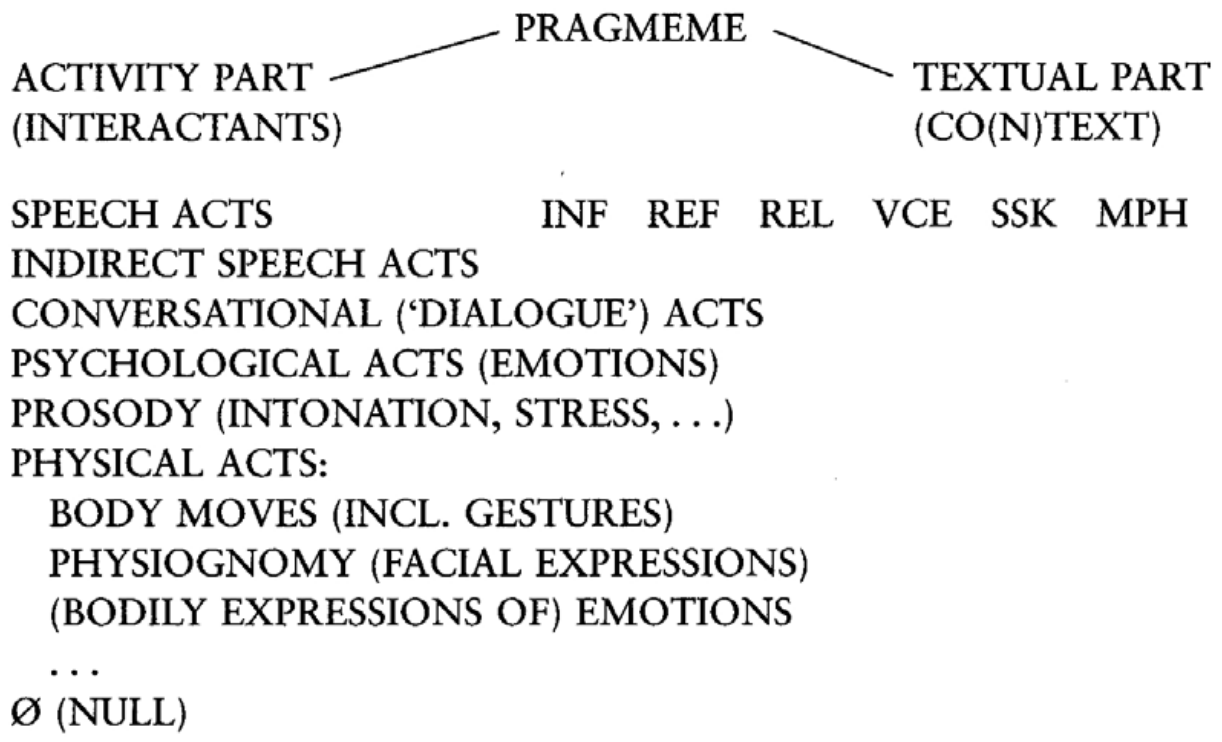

PRACT

ALLOPRACT

PRAGMEME, PRACT, ALLOPRACT

Figure 1: Mey’s Scheme of a Pragmeme

The activity part covers speech acts, indirect speech acts, conversational ('dialogue') acts, psychological acts, prosodic acts and physical acts. The textual part involves context elements: INF representing "inference"; REF, "Reference", REL, "relevance"; VCE, "voice"; SSK, "shared situation knowledge"; MPH, "metaphor"; and M "metapragramatic joker". These can be illustrated with the expression, "The card [case note] is for the woman with natural document", made by a doctor to a nurse when detailing the latter about the treatment course to follow in the presence of other clients. By the term "natural document", reference is made to an obese woman; a possible inference is the doctor's goal to save the client's face from the embarrassment that may emerge from an unshielded choice such as "obese" or "very fat"; relevance occurs in the doctor's addressing himself to both the nurse's seeking of direction and the sensitivity of exposing the client to stigmatisation; the voice or perspective is medical and sociocultural as it represents the institution of medicine's and the Nigerian or Yoruba society's strategic euphemisation; the knowledge of the selection and the connection with the situation is shared between the doctor and the nurse. The union of the activity and textual parts produces a pract or an allopract which is an instantiation of a 
pragmatic act. "Every pract is at the same time an allopract...." (Mey 2001: 221). For example, in the concealment context in Nigerian hospitals, the expression "You will go for 333 screening" (You will go for HIV/AIDS laboratory examination) practs veiling as will be shown later in the analysis, demonstrating a synthesis of indirect speech acts (activity part) and (con) textual features (Reference, Inference, Voice and Shared Situation Knowledge). In the words of Tseng (2010: 1986), the scheme of pragmatic acts proves useful in that it "broadens our view of what acts may be performed by language..., the notion is an extension of speech act theory in that it is not constrained by rules of grammar... The scheme of the pragmeme is flexible since it is based not on rules but predicated on choices".

Mey (2001) proposes situated speech acts i.e. practs or allopracts which "rely on, and actively create, the situations in which they are realised" (Mey 2001: 219). Their realisation, according to Mey, shares borders with concepts of speech events in ethnography and anthropology. In institutionalised activities such as teaching and doctor-patient interactions, for example, speech is restricted:

Only certain utterances can be expected and will thus be acceptable; conversely the participants in the situation, by their acceptance of their own and others' utterances, establish and reaffirm the social situations, in which they find themselves as utterers (Mey 2001: 219).

Speech acts in this context have been described as pragmemes by Mey.

According to Capone (2005: 1357), the goal of pragmeme as speech act is to ensure "effects that modify a situation and change the role of participants within it or bring about other types of effects such as exchanging/assessing information, producing social gratification or otherwise, rights and obligations and social bonds". As a consequence, a pragmatic act is performed which involves adapting oneself to a context as well as adapting the context to oneself (Mey 2001; Odebunmi 2006: 157; Keskes 2010). Thus, language and society rules combine in dictating meanings which synchronise with the context of culture in which speech occurs.

Capone (2005: 1357) identifies three embedding types associated with pragmeme:

the embedding of an utterance in the context of use, with an aim to determine the referential anchors that complete the propositional form of the utterance; the embedding in rules that systematically transform whatever gets said in a context into whatever is meant there; in conformity with the social constraints and rules bearing on the utterance in question; the embedding in the context whose features are transferred onto the utterance by eliminating semantic or otherwise interpretative ambiguities and enriching further its (range of) interpretations, by making them more specific.

The first embedding captures the lexical and referential configuration within a situational context; the second ensures a transformation from propositions to meaning through observance of interactional rules; and the third provides contextual aids that specify speaker goals and intended interpretations. All these embedding types are relevant to doctor-client interactions with respect to concealment as will be demonstrated in Section 5 below. Both the referential anchors of linguistic elements and their context-aided meanings achieved within the goal of concealment are important in coming to terms with news management in the Nigerian hospital. 
Pragmemes essentially consist in transformations (Mey 2001) in that they redirect or change the original orientation of an illocutionary force by imposing contextrestrained meaning. These transformations, in the words of Capone (2005: 360), "are based on a number of principled and conventional interactions between utterance meaning and certain contextual and situational configurations" (cf. Levinson 1979). When aligned to the discourse of concealment in the Nigerian hospital, an expression such as "You are zero positive", which, means the patient is HIV positive, when interpreted, using the pragmemic provision of INF (Inference), transforms from its conventional referential configuration to reference to a terminal prognosis. This is supported both by the institutional environment of the hospital in which it is said and by local interactional factors which include the ailment, previous interactions on the ailment, current condition of the client and the nature of the exchange in which the announcement is made.

\section{Analysis and findings}

My analysis is done at two operational levels as shown below:

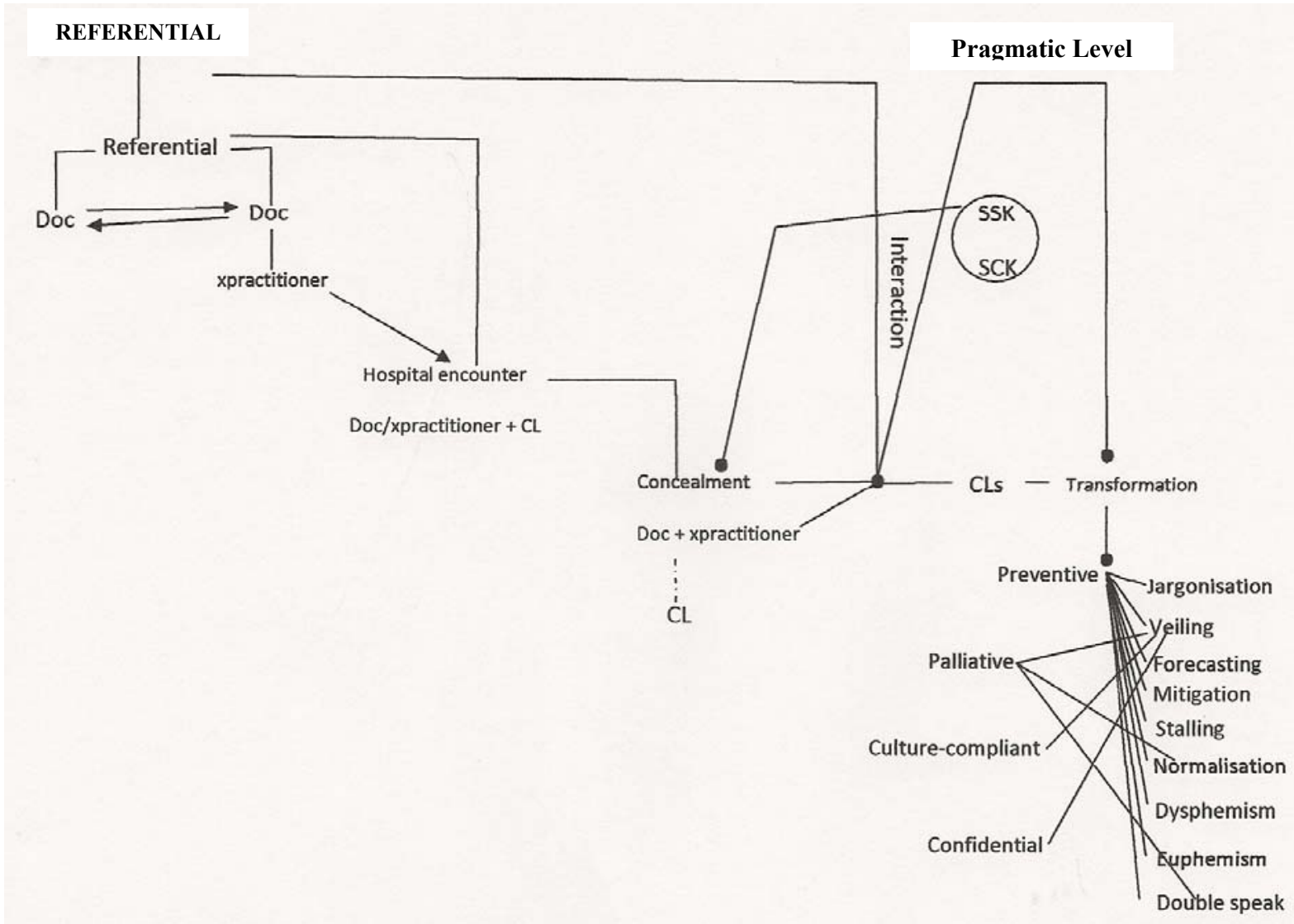

Figure 2: Concealment in Consultative interaction

The chart shows a broad, two-stage operation: REFERENTIAL level and pragmatic level. In more specific terms, it demonstrates Capone's (2006) three types of embedding in large measure, including Mey's transformations, and combines some other features of pragmemes and medical discourse. 
At the REFERENTIAL level, all the words which are used in concealment by doctors have referential values, pointing to certain diseases, medical procedures or hospital activities known to doctors and, to a certain extent, other members of the medical team (e.g. nurses, laboratory technologists, etc). These referential items are employed by doctors (and sometimes by other members of the medical team) in hospital interactions in which clients may or may not be involved. At this point, concealment is not involved as objects, actions and experiences have to be described to correctly situate the attention required by the client (henceforth "CL"). Concealment comes into the picture at the pragmatic level when doctors (sometimes in conjunction with other members of the team, or the client) consider it necessary to keep certain information from a client, other clients or their relations. This means that concealment in the Nigerian hospital is strictly a pragmatic act as it is performed only selectively.

When the need for concealment is established, the referential elements have to interact with both the context and the goals of users to be realised as concealment items. Context here connects with the goals of concealment operators through SSK (Shared Social Knowledge) (Mey 2001) and SCK (Shared Cultural Knowledge) (Odebunmi 2006c). These presupposition features bring concealment items into the communication with the clients (and relations), leading to transformations, i.e. context-imposed meanings. These transformations are preventive (realised through nine strategies), palliative (realised through three strategies), culture-compliant (realised through only one strategy) and confidential (realised through only one strategy). As said variously (cf. Odebunmi 2003, 2005, 2006b, 2007, 2008, 2010c), and as will be seen in the examples below, communication in the Nigerian hospital, especially between doctors and patients, takes place in Standard British/American English, Nigerian indigenous languages, Nigerian English and Pidgin English. I refer my readers to my publications, especially Odebunmi (2006b, 2008 and 2010d) for details on how and when the languages are used in the hospital. In the examples that follow, only two codes, English and Yoruba are used. Yoruba is in bold fonts while English is in regular fonts.

\subsection{Referential level}

At the REFERENTIAL level, all lexical items in the interactions, which are equally capable of being used for concealment at the pragmatic level, perform mere descriptive functions. This dimension has institutional and social bases. The institutional basis restricts itself to the knowledge doctors (and other members of the team) have of terms to describe a particular disease or medical procedure. Therefore, when not interacting with clients, the items circulate only within the institutional orbit. The social scope of the terms comes alive when doctors have to relate with clients and provide descriptions of the object of their visits.

The two situations below can be examined:

Ex. 1:

$\rightarrow$ Doctor: Get the laboratory ready for $C S$ now=

Nurse: $\quad$ Okay sir.

Ex 2:

$\rightarrow$ Doctor: $\quad$ Madam, you see, (0.2) we have to do CS for your daughter (.)

Client: $\quad$ Ah, MY GOD; why? 
In Ex. 1, the doctor (henceforth "CL") talks to a member of the medical team who needs no explanation for the procedure being instructed about, i.e. "CS" (Caesarean operation). This is institutional. At the social level, in Ex. 2, the doctor tells the mother of a pregnant woman that her daughter would have to undergo "CS" without explaining to her what the term means. His assumption that the client would have no difficulty understanding the term is confirmed when the woman exclaims in fear. At this level, no concealment is involved as the term is used to pick out a medical procedure which each of the hearers is able to place correctly. Given that the doctor needs the consent of the client's mother, no concealment is warranted in the first place.

\subsection{Pragmatic level}

At the pragmatic level 25 diseases/medical procedures were concealed by doctors (sometimes in conjunction with clients) to achieve four goals or transformations, namely, preventive, palliative, culture-compliant and confidential. Meanwhile, it is essential to note that concealment in the interactions hinges on six factors: doctors' previous experiences with clients with similar cases and how such clients have reacted; knowledge of clients' emotional states i.e. their average reactions to socio-medical issues; common grounds with clients; cultural scripts and doxas i.e. influences of religion and culture; phase factor i.e. the stage of the disease or procedure being dealt with; and others factor i.e. the presence of a third party.

\subsubsection{Concealed diseases/procedures:}

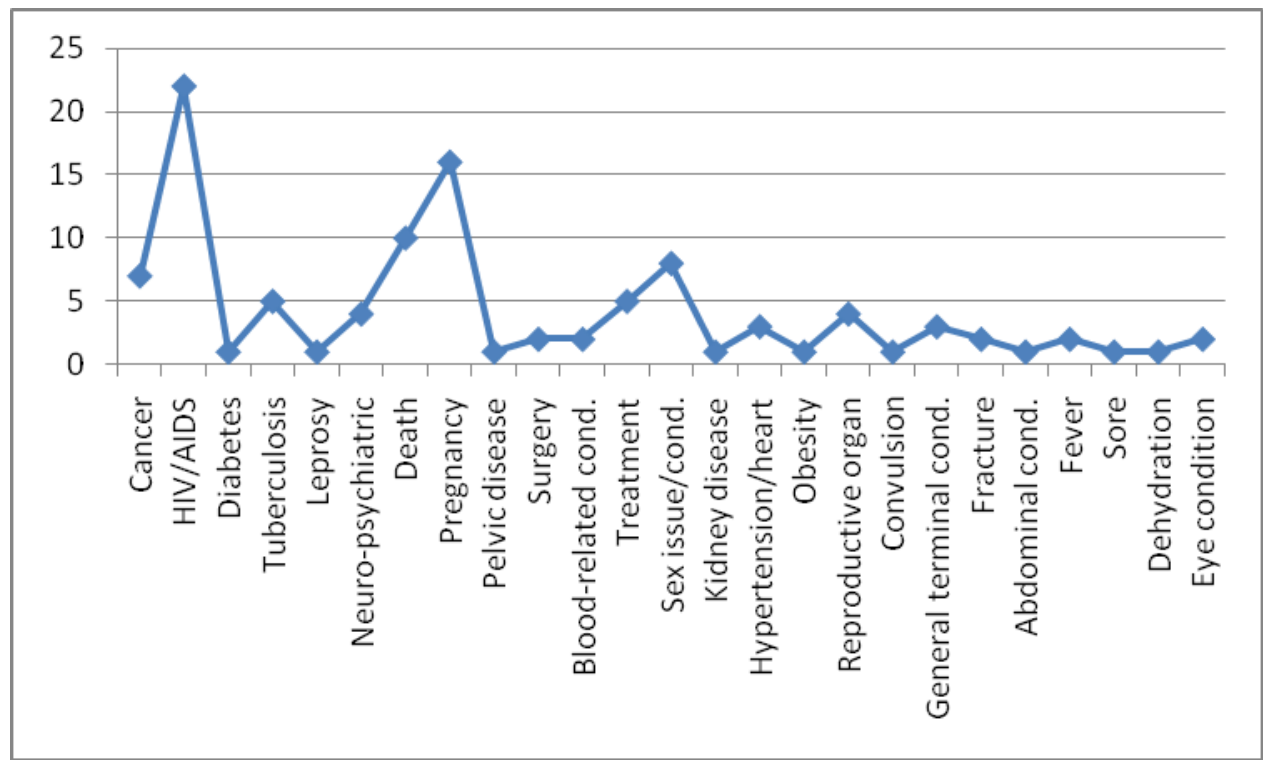

Figure 3: A line graph showing the frequency distribution of concealed conditions/ procedures 
Figure 3 shows that "HIV/AIDS" takes the lead. Next is "pregnancy" which is followed by death. Others follow in this order: "sex issue or condition", "cancer", "tuberculosis"/"treatment"; "neuro-psychiatric condition"/ "reproductive organ"; "hypertension or heart disease"/"general terminal disease"; "surgery"/"blood-related condition"/"fracture"/“fever"//eye condition"; and "diabetes"/"leprosy"/ "pelvic disease"/"kidney disease"/"obesity/convulsion"/"abdominal condition"/ "sore" and "dehydration".

That HIV/AIDS has the highest frequency confirms its global rating as a leading world killer disease. Added to this impression are the questions of heterogeneity and stigma. That pregnancy is concealed is a socio-cultural issue. First, because of the high value placed on procreation in Nigeria (and in Africa in general), child bearing is treated with sacred importance. Until pregnancy is advanced enough for the public to see, many married women in South-western Nigeria do not want to talk about it. Second, given that many pregnancies in South-western Nigeria are teenage, pre-marital or extramarital experiences, bearers prefer to talk indirectly and secretly about them. Also, the belief that witches or individuals with evil intents, especially in a polygamous context, are believed to attack the foetus supernaturally, encourage concealment. That death is concealed at a significant level shows the Yoruba's high emotional bond with loved ones which often makes direct announcements of death news a cautious task especially if the deceased is young.

Sex-related conditions also affiliate with cultural doxas as many Yoruba people prefer sex to be discussed with camouflages. Cancer is simply feared. Its relatively lower occurrence points to its inevitability, which has made it to be accepted, many times, as a matter of course, unlike HIV/AIDS which is linked with heterosexuality. Other conditions are concealed at a relatively low level, because they are either not very common experiences or are not considered sufficiently deserving concealment. Table 1 below shows the conditions/procedures, examples of terms/items used to conceal them and their percentage distribution:

\begin{tabular}{|l|l|l|l|}
\hline $\mathbf{s} / \mathbf{n}$ & Concealed Conditions/Procedures & Examples & $\begin{array}{l}\text { Percentage } \\
\text { Distribution }\end{array}$ \\
\hline 1. & HIV/AIDS & 333, ARC & 20.75 \\
\hline 2. & Pregnancy & MR, PT & 15.09 \\
\hline 3. & $\begin{array}{l}\text { Death } \\
\text { Sex-related issues or condition }\end{array}$ & $\begin{array}{l}\text { Kaput, GO } \\
\text { G-trace (gonorrhoea), sleep } \\
\text { with (have sex with) }\end{array}$ & $\begin{array}{l}9.52 \\
7.55\end{array}$ \\
\hline 5. & Cancer & Bad ulcer, C.A & 6.60 \\
\hline 6. & Tuberculosis, treatment & Kochs; CQ (chloroquine) & 4.72 \\
\hline 7. & $\begin{array}{l}\text { Neuro-psychiatric condition; } \\
\text { reproductive organ }\end{array}$ & Ward T; VE & 3.77 \\
\hline 8. & $\begin{array}{l}\text { Hypertension or heart disease; general } \\
\text { terminal disease }\end{array}$ & $\begin{array}{l}\text { HTP; ...wait for the next 24 } \\
\text { hours }\end{array}$ & 2.83 \\
\hline 9. & $\begin{array}{l}\text { Surgery; blood-related condition; } \\
\text { fracture; fever; eye condition }\end{array}$ & OP; PCV; broken stick; CAT & 1.87 \\
\hline 10. & $\begin{array}{l}\text { Diabetes; leprosy; pelvic disease; } \\
\text { kidney disease; obesity; convulsion; } \\
\text { abdominal condition; sore; dehydration }\end{array}$ & $\begin{array}{l}\text { DM; Hansen's disease; } \\
\text { EUA; } \text { urea; natural } \\
\text { document; praising God; any } \\
\text { parent around; bad egg; dry. }\end{array}$ & 0.94 \\
\hline
\end{tabular}

Table 1: Concealed Conditions/Procedures 


\subsubsection{Goals and strategies}

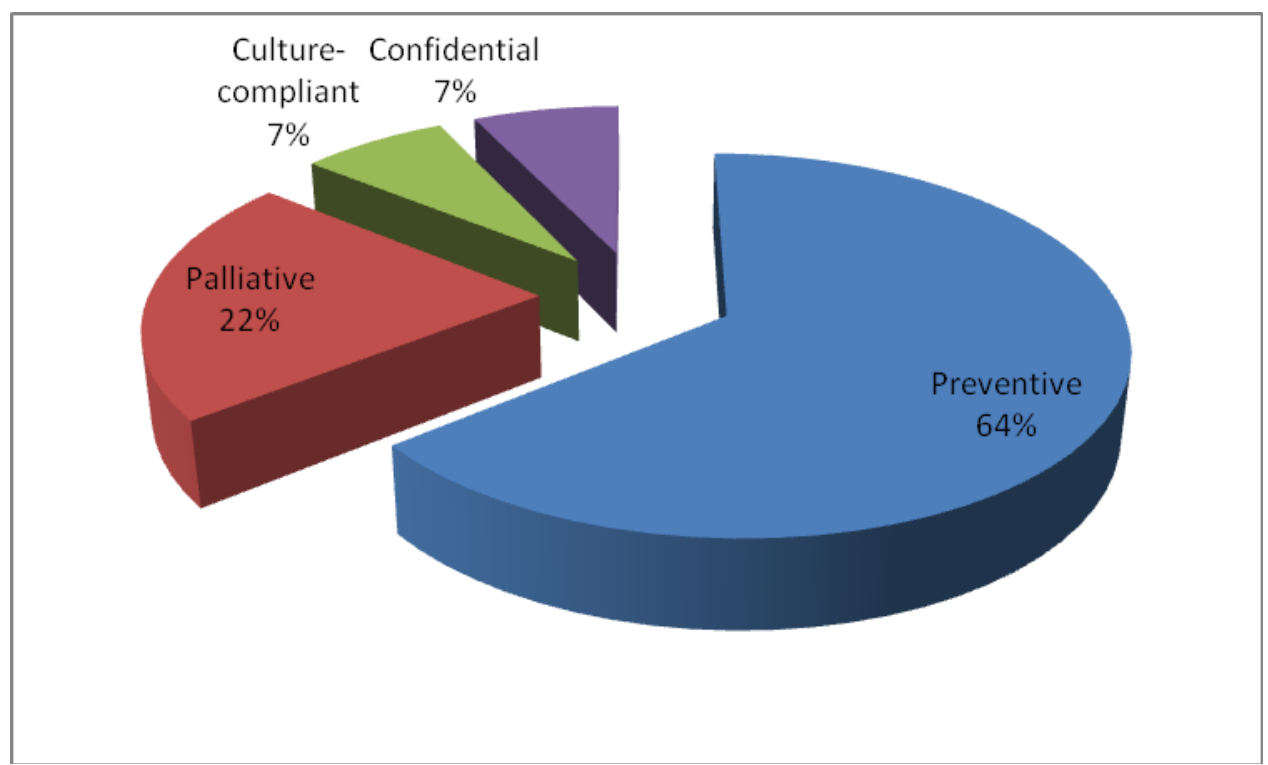

Figure 4: Goals

The preventive goal predominates with $64 \%$. This indicates, as will be shown shortly, that much of concealment communication attempts to forestall "crisis situations" in the hospital. The palliative goal comes next with $22 \%$. Culture-compliant and confidential goals come last with $7 \%$ each.

The preventive goal is concerned with the doctor's agenda to bar, on CL's part: knowledge of their conditions or hospital facilities, fear, doubt, self-prescription, and stigmatisation. The palliative goal, which appeals to temporary relief by cutting down the psychological burden of CL, strives to avoid pandemonium in the hospital and a worsening of CL's condition. The culture-compliant goal enlists with the people's cultural (and sometimes religious) doxas. The confidential goal works with medical ethics which restricts the doctor's freedom with information relating to CL's condition. 


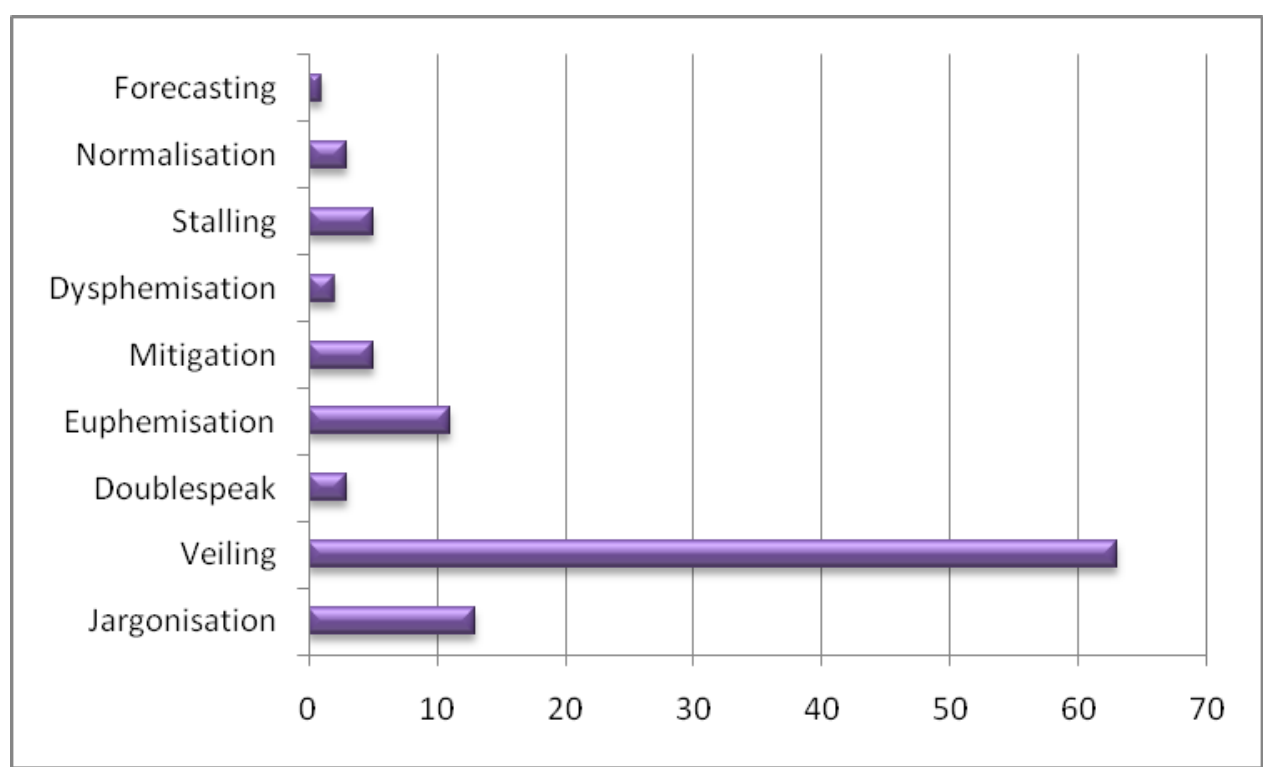

Figure 5: Concealment Strategies

Veiling has the highest representation, which points to the fact that much of the concealment communication is directed towards transmitting unclear information to CL. It is conceived of, in this research, as short-cuts employed by doctors to shroud an ailment or medical procedure. Jargonisation, which comes next, works on the assumption that CL lacks knowledge of the technical lexemes of medicine, which Doc, in the context of concealment brings into the interaction with no simplified reformulations. It thus captures doctors' use of exclusively medical lexemes in communicating with clients (or their relations) to keep away certain information from them. Such terms are employed in situations where doctors are absolutely sure, given their knowledge of clients' socio-professional background, that clients have no access to the terms, a reckoning that sometimes fails with devastating consequences, as will be seen in Section 6 below. Euphemisation simplifies CL's condition by minimising the harshness or unacceptability of negative diagnoses or contextually dispreferred news. Stalling indicates withholding information from CL either by silences or avoiding diagnosis-related news. Mitigations are hedges which deflect the full magnitude of a diagnosis. Normalisation is a strategy by which a mild part of a diagnosis is presented to the client to give the appearance of ordinariness. Doublespeak offers two possible interpretations or options without specifying which is locally preferred by the doctor. Dysphemisation replaces the original medical name of an ailment with a non-mitigated, face-threatening lexical choice in diagnostic news. In the context of concealment, it subtly castigates or piques the client, and simultaneously saves his/her face considering others factor. Forecasting is a pragmatic tool employed by doctors to provide some clues about a diagnosis or condition and rouse the interest, anxiety or suspicion of the client or their relation (s). 


\begin{tabular}{|l|l|l|}
\hline $\mathbf{s} / \mathbf{n}$ & Goals & Strategies \\
\hline 1. & Preventive & Jargonisation \\
& & Veiling \\
& & Forecasting \\
& & Mitigating \\
& & Stalling \\
& & Normalisation \\
& & Dysphemisation \\
& & Euphemisation \\
\hline 2. & Palliative & Doublespeak \\
& & Veiling \\
& & Normalisation \\
\hline 3. & Culture-compliant & Doublespeak \\
\hline 4. & Confidential & Veiling \\
\hline
\end{tabular}

Table 2: Goals and Strategies

As Table 2 shows, all the nine strategies are used in preventive goals; three (veiling, normalisation and doublespeak) in palliative goals, two (veiling and euphemisation) in culture-compliant goals and one (veiling) in confidential goals.

\subsubsection{Preventive goals}

Doctors' preventive goals rely on jargonisation, veiling, forecasting, mitigation, stalling, normalisation, dysphemisation, euphemisation and doublespeak. Jargonisation in concealment utilises non-popular medical terms which trifurcate into:

(i) strictly technical terms (e.g. "urea" for "kidney");

(ii) proper names (e.g. "Hansen's disease" for "leprosy");

(iii) new medical terms (e.g. "retroviral infection" for "HIV/AIDS").

The short conversation below explains the preventive programme clearly:

Ex 3:

$\rightarrow$ Doctor: $\quad$ Madam, the condition, I suspect, is retroviral infection=

Patient: $\quad$ Okay (.)

Doctor: $\quad$ You will go to the lab now, for us to be sure=

Patient: Thank you, doctor

Doc in Ex. 3 would not ordinarily announce the diagnosis of HIV/AIDS the way he has done in this interaction if he lacks knowledge of CL's socio-professional background. He simply works on CL's ignorance of the semantic shades of the term, "retroviral infection" (a relatively new medical term). CL does not demonstrate any knowledge of the reference identified by the doctor's 'retroviral infection' as she readily agrees with the doctor on his observation (by saying "okay") and expresses appreciation for the doctor's service (by saying "Thank you, doctor"). Her turn speed which leaves no gap between her speech and that of the doctor further confirms that she has no inkling of the semantic import of the term. The doctor is contextually restrained from the blunt 
"HIV/AIDS", which is already popularly distributed, because it could generate negative emotional reactions from CL. Thus, this pragmatic act supports Mey's (2001:43) assertion that "context determines what one can say and what one cannot say"

Jargonisation is sometimes employed to prevent clients' doubts of doctors' competence. The only class of lexemes popular in this regard is strictly technical terms.

Ex 4:

Patient: $\quad$ Doctor, malaria yen o lọ o

(Doctor malaria that not go)

(Doctor, the malaria is yet to be cured)

Doctor: $\quad$ Alagba, e ma worry (0.6)

(Gentleman, you don't worry)

(Gentleman, don't worry)

$\rightarrow$ A ma treat plasmodiasis (0.2)

(We shall treat plasmodiasis)

Patient: $\quad O$ daa. Se ngo sa gbadun?

(It good. Will I just well?)

(Alright, will I be well?)

In Ex. 4, the doctor uses the term "plasmodiasis", a technical synonym for "malaria fever" as a metareferential token to describe the malaria fever experience of CL. As gathered from the interview conducted with the doctor, he had the intention to deny CL access to the object of his reference. He succeeds in achieving this goal because CL does not challenge his new diagnosis. Rather, he submits to the doctor's authority provided his new procedure would fetch him healing:

Doctor: $\quad$ Alagba, e ma worry (0.6)

(Gentleman, you don't worry)

(Gentleman, don't worry)

$\rightarrow$ A ma treat plasmodiasis $(0.2)$

(We shall treat plasmodiasis)

Patient: $\quad O$ daa. Se ngo sa gbadun?

(It good. Will I just well?)

(Alright, will I be well?)

"Plasmodiasis", an exclusively medical term, is used in a Yoruba conversation with a client who understands little or no English and who, at best, could understand "malaria" or "fever", which are often used alternatively, but rarely as a single word, among the half-literate or illiterate. That the word is beyond the limits of CL's English lexicon is demonstrated in his response:

Patient: .... Se ngo sa gbadun?

(It good. Will I just well?)

(Alright, will I be well?)

which admits Doc's therapy proposal by the use of the acceptance-indicative adjective "O daa" (alright), and which drifts along with Doc's treatment direction by the situation-admittance interrogative, "Se ngo sa gbadun?" (Will I be well), contextually 
implying "whatever you say, provided it will give me healing, I don't mind". In a way, Doc's concealment strategy has influenced CL's treatment option without giving him a voice in the decision process, thus establishing that except where clients are informed beforehand of the use of concealment items, the discourse is a uni-party or unilateral choice made only on Doc's decision. When asked why he opted for the isosemantic "plasmodiasis", the doctor expressed the opinion that it was safer to talk technically and becloud the condition than to allow CL to conclude that he lacked the competence to treat "ordinary malaria fever", which might mar his professional profile. The argument of information access denial was also advanced by other doctors with respect to preventing self-prescription, which constituted another ground on which jargonisation was employed to conceal information. Given that malaria fever, a tropical disease, is prevalent South-western Nigeria, many CLs, often after being treated once in the hospital, would go and buy the prescribed drugs during subsequent episodes without doctors' prescriptions. One measure to prevent this is for the doctor to employ isosemantic diagnostic items which will be difficult for CLs to place correctly, thus stopping them from self-prescription.

Many veils employed by doctors are simple and complex abbronyms (Odebunmi 1996, 2006, 2010c). The term "abbronymy" (abbreviation + acronym) was first used in Odebunmi (1996) to refer to letter representations of full word forms which may or may not be pronounceable. Odebunmi (1996) identifies simple and complex abbronyms in scientific texts. While the former is predictable from the full form (e.g. "UN" for "United Nations"), the latter is not (e.g. "CQ" for "chloroquine").

From the accounts of the doctors, all the simple abbronyms for veiling (DM, VD, ARC, UTI, IUD and D \& C) are employed to "hide the ailment from the client", and "for other clients not to notice or know." These present two situations: (i) where CL is not aware of his/her condition; (ii) where CL is aware of his/her condition. In (i), abbronyms such as DM (Diabetes Mellitus) and VD (venereal disease) are used. In (ii), ARC (AIDS-related complex), UTI (urinary tract infection), and D \& C (dilatation and curettage - abortion) are engaged. The use of items from the two groups is contingent on the common ground explored. An interactional example can be cited:

Ex. 5:

Patient: $\quad$ Doctor, mo ti de (0.3)

(Doctor, I have come)

Doctor, I am around)

Doctor: $\quad$ O daa, Davi::d ((calling a nurse $))=$

(It good)

(Okay)

Nurse: $\quad$ Sir: (.)

$\rightarrow$ Doctor: $\quad \mathrm{D}$ and $\mathrm{C}=$

Nurse: $\quad$ Okay sir

I was present at the interaction in Ex. 5, which took place at a lobby in front of the doctor's consulting room. The doctor shares the common ground on the meaning of " $\mathrm{D}$ $\&$ C" for "abortion" with the nurse and the client. He would attract unpleasant reactions if he had opted for a popular expression such as, "Nurse, this woman is for abortion; go and attend to her". The nurse simply accepts the doctor's order with "Okay, sir", taking into account others factor. None of the other clients present at the scene expressed 
negative feelings despite the unacceptability of abortion in the Nigerian society, which announces the achievement of the preventive goal. The belief on which the concealment is built is initiated by the CL when she says, "Mo ti de" (I am around), which suggests a prior arrangement outside the immediate consultative environment. She generates an implicature by relying on Doc's ability to saturate her underdeterminate proposition, which does not submit the actual object of her visit i.e. an abortion. Working within the ambits of her assumption, Doc cognitively enriches and processes her proposition by acknowledging her arrival and evoking their shared belief by the utterance of "okay", and invites the nurse into the medical activity required on a professional rather than a social assumption, the kind he operates with CL.

The complex abbronyms, like some simple ones, are used to "hide medical procedures or ailments from clients or others". Beyond this, they are employed to mystify some ailments' original known terms to make mappings impossible by clients. Items such as "CA" (cancer), "OP" (operation), "CQ" (chloroquine), "TB" (tuberculosis) etc, fall in the first category. The items are formed by a selection of the first letter of every one of the words, and another letter arbitrarily selected from the full forms. "CQ" occurred in the following interaction:

Ex 6:

$\rightarrow$ Doctor: $\quad$ Give him i.m CQ c.c stat $=$ ((Leaves))

Nurse: $\quad$ Alright sir. (0.5)

Patient: $\quad$ I hope that is not chloroquine? --

Nurse: $\quad$ There is no problem; you will be alright.

The choice of "CQ" has been used pragmatically constrained in Ex. 6. CL is reactive to chloroquine, about which he had formally complained to the doctor. Yet, Doc, from my interaction with him after the procedure, judged chloroquine (CQ) the best medicine for CL's condition. Doc, in the interaction, pushes his preventive goal through by selecting a veil, "CQ", which is shrouded by co-textual technical tokens: "i.m", "cc" and "stat". CL's suspicion, carried by "I hope that is not chloroquine?" is suppressed by the nurse's appeal to Pollyanna principle, which perhaps makes CL to take the treatment without further questions. In this interaction, the preventive bid of Doc almost failed despite the calculated and monitored usage of "CQ" whose link with the original term, "chloroquine", CL suspected, given his level of education and shared background with Doc on his preferred treatment mode. When he says, "I hope that is not chloroquine", he evokes his limited knowledge of medical jargon and seems to move to resist the administration of chloroquine, but he could not sustain this effort because of the nurse's smart intervention which stalls further information course.

Where complex abbronyms are used to mystify the ailment's original known terms, doctors have had practical necessities to devise new concealment strategies. One of the doctors interviewed said that "HIV" used to be the term for concealing HIV/AIDS infection in his hospital until it became very popular, and clients were declining being tested for it. Consequently, the term "VT" had to be created to refer to it. The new coinage, according to him, had positive reception because it was not linked with "HIV" by clients.

Three diseases or conditions are mystified in the hospitals: HIV/AIDS (realised as VT, IHV, RVS, RVST), hypertension/high blood pressure (realised as HTP, HBP), 
and tuberculosis (realised as TBS). The abbronyms have been formed through reordering (e.g. IHV, HTP: HIV, HPT), arbitrary selection of letters (e.g. VT = (retroviral infection) and addition of new letters to existing abbronyms (e.g. $\underline{H B P}, \mathrm{HP} \underline{\mathrm{S}}$, RV $\underline{\mathrm{S}}$, RV $\underline{\text { ST)}}$. No interactional usage of the items was found in the data, but some doctors gave instances such as:

$\rightarrow$ Ex. 7: $\quad$ You will do test for RVS

$\rightarrow$ Ex. 8: $\quad$ It's $H T P$; it will be controlled.

Ex. 7 was said by a doctor, in his consulting room, to a client who was suspected to be HIV positive. According to the doctor, the client displayed no knowledge of the abbronym and the condition for which a laboratory examination was being ordered. Ex. 8 was said as a response to a client's questions: "Is it hypertension? Wouldn't it kill me?" In this case, the doctor has dispreferred "BP" which CL was expected to know. His choice of HTP was to mystify the old term to be able to gain the co-operation of CL who was already expressing doubts about survival. The client's flooding out response (Maynard 2003): "Is it hypertension; wouldn't it kill me?", strictly constrains the choice of a veil, itself supported by the institutional context where "only certain utterances can be expected, and will thus be acceptable" (Mey 2001:219).

Mitigations dwelling largely on predictives are employed to avoid stating the exact truth of CL's condition (e.g. "You have 50-50 chance" when announcing a cancer prognosis). Stalling, which engages interpretives and predictives avoid announcing diagnoses and observations (e.g. "That means you are not pregnant" said to conclude Doc's examination of a CL who presents with fever and body pains). Normalisation which works with assertives keeps details of CLs' condition from them or from their relatives (e.g. "you have toilet infection" referring to "yeast infection" and "The patient is unco-operative" referring to a CL who could not be revived from coma). In these two cases, the conditions are made so ordinary that CLs or relations could hardly be alarmed by them, but they could be pressured to make incorrect inferences. According to Maynard (1996: 119-120), "for one, using euphemism as a way of stalling can lead to a recipient to believe something different from the actual state of affairs....Another form of incorrect inferencing occurs because in the face of stalling, a potential recipient who senses something is amiss is invited to make guesses". My data contain testimonies of clients exploiting the former option when doctors deploy normalisation, and the latter when they employ stalling.

Euphemisation works with extension (e.g. "evacuation" for "abortion"), borrowing (e.g. "slim disease" - borrowed from East African English - for "HIV/AIDS"), slang (e.g. "mud" for "death"), Nigerianisms (e.g. "take-in" for "being pregnant"), and downtowners (e.g. "The patient is disturbed" for "a psychotic patient"). Doublespeak appears as interjectives (e.g. "Oh! God" for "Gonorrhoea"), coinage (e.g. "triple cross" for "HIV/AIDS") and ambiguity (e.g. "pack" for "dead"). They are respectively employed to trivialise CL's condition, keep information from CL and hide information from others. One interactional example of stalling can be cited here:

Ex. 9:

Patient's father: Doctor, good morning sir $=\mathrm{I}$ am the father of the child admitted in Ped for three days now= Please sir, any hope? =

(Pediatric ward) 
$>$ I want to know our fate sir $<$. We came from Oyo to Ogbomoso.

We have spent two days at a private hospital before we came here.

Any hope $\operatorname{sir} \downarrow$

$(0.4)$

Doctor: $\quad$ Let me see the child.

(The doctor, the father and the relation go to the ward)

Patient's father: ${ }^{\circ}$ What is our fate sir $^{0}$ ?

Doctor: (examines the boy on the bed)

Patient's father: ${ }^{\circ}$ What's our fate ${ }^{0}$ ?

Doctor: ((silent)).

$(0.5)$

$\rightarrow \quad$ Okay; ${ }^{\circ}$ let's wait for the next twenty-four hours ${ }^{\circ}$. ((THE BOY DIED TWO HOURS LATER))

The father's "What is our fate sir?" receives no verbal response from Doc who prefers medical exactitude which could only be obtained through examinations as he does shortly after the question. After the examination, the father repeats his question, which the doctor could not avoid answering after he has performed the necessary physical examination. Doc's long silence followed by the transition marker "Okay" and the predictive "Let's wait for the next twenty-four hours" imply bad news. From his equivocality and CL's eventual death, it is obvious that Doc decidedly stalls the news to prevent pandemonium in the hospital, thus confirming Maynard's (1996: 119) view: "stalling implies that there is bad news to tell, but those who are 'in the know' and are potential deliverers avoid the telling".

On certain occasions, appeal is made to concealment to prevent fear and stigmatisation. Veiling and dysphemisation are common pragmatic tools to prevent stigmatisation while forecasting, mitigation and veiling are engaged to prevent fear. Where veils are used to prevent stigmatisation, abbronymy and coinage are drawn upon. Examples include: "WWG" to refer to "psychotic patients. "WWG" picks out "West West Wing", a ward for mental patients; "natural document" (obesity), a coinage, is used to save CL's face as obesity is almost universally stigmatised. Dysphemisation which relies on indirectness (e.g. "illness that affects population" for "gonorrhoea") and metaphor (e.g. "bad eggs" for "deep sore") is used to save CL's face when reference has to be made to their condition in the presence of others.

Fear is prevented through forecasting which employs hinting as a major tool (Maynard 1996). One of the respondent clients recounted the following conversation she had with a doctor:

Ex. 10:

Doctor: $\quad$ Mary =

Patient: $\quad$ Sir (0.2)

$\rightarrow$ Doctor: $\quad$ Do you have any of your parents here with you or your relation? (.)

Patient: $\quad$ Any problem? (.)

$\rightarrow$ Doctor: $\quad$ No 
CL (Mary), in Ex. 10, in an interview, said that the doctor did not announce her hernia diagnosis until her father came to the hospital. From her account, the doctor had to temporarily stall the diagnosis, through forecasting, because the doctor held a script of her emotional reaction being a regular visitor at the hospital. The conversational sequence is shaped for the pragmatic act. Calling Mary's name occurs after history taking and preliminary examinations. What is stalled is diagnostic communication (Heritage and Maynard 2006). In the Yoruba Culture, such name calling is strategic as it provides footing change to a solemn subject. The doctor's verbal action thus aligns with the cultural doxas of the Yoruba (cf Odebunmi and Auer 2011). Mary's speedy response establishes her eagerness to take the news already signalled, but Doc slows down the tempo to allow her settle down fully for the information. Mary's cultural background and knowledge of the medical institutional script relating to bad news prompt her to, after a very brief pause, probe into Doc's request, with an equal interrogative: "Any problem?" The doctor's refutation notwithstanding, there is sufficient evidence that his forecasting goal has been achieved.

When veiling is used to prevent fear, simple abbronyms are preferred. Many of our doctor respondents cited "CS" (Caesarean operation) as a term whose implications often go unnoticed among many of their clients and their relations, except those who had undergone the surgery or some educated ones. Sometimes, serious conditions are mitigated to prevent CL nursing fear about them. Metaphor and understatement are major discourse tools employed here. During a consultative session, a deep fracture was simply referred to as "a broken stick" which tempered the gravity of the condition and consequently dowsed the deep fright already being expressed by CL. In another interaction, in another hospital, "pale" was used to describe a serious anaemic condition. We can examine the interactional context in which this occurred:

Ex. 11:

Doc: Se iyen naa ni?

(Is it that only is?)

(Is it only that?)

Pat: Un

(Yes)

Doc: Se mo so fun yin pe blood yin loo le?

(Is it I told for you that blood you goes down)

(Did I tell you your blood level is low?)

So, e maa ni lati mura si tonic yin. Se e de nlo daadaa?

(So, you will have to concentrate to tonic your. Is it you are using it well?)

(So, you will have to use your tonic consistently. Are you using it well?)

Pat: Beeni

(Yes).

Doc: Yato si complain pe ito yin yellow,

(Apart to complain that urine your yellow),

(Apart from the complaint that your urine is yellow),

se ko de si any other complain min?

(Is it not there is any other complaint any?)

(Do you think there is no other complaint?)

Pat: Then, naa, ori maa ntun nfo mi, otutu.

(Then, again head is aching me, cold). 
(Then, I also have headache and cold).

Doc: Un, nigba wo ni otutu yen ti bere?

(Yes; when did cold that has started?)

(Yes; since when has the cold started?)

Pat: Last week.

$\rightarrow$ Doc:Oju yin si pale. Ounje nko?

(Eyes your still pale. Food what about?)

(Your eyes are still pale. Do you eat?)

Pat: Mo njeun.

(I am eating).

(I eat).

In Ex. 11, the consultation centres on CL's pregnancy experience. CL volunteers information about her improving condition and her coloured urine in her narrative excluded from the transcript, but the doctor personally observes that her blood level is too low. CL confirms this in her CI (condition-specific information (Odebunmi, 2010c)

11 (i):

Doc: Se mo so fun yin pe blood yin loo le?

(Is it I told for you that blood you goes down)

(Did I tell you your blood level is low?)

So, e maa ni lati mura si tonic yin. Se e de nlo daadaa?

(So, you will have to concentrate to tonic your. Is it you are using it well?)

(So, you will have to use your tonic consistently. Are you using it well?)

Pat: Beeni

(Yes).

It takes the doctor's further prompting to get CL to provide further CIs which eventually point out a major health problem of CL's:

Ex.11 (ii):

Doc: Yato si complain pe ito yin yellow,

(Apart to complain that urine your yellow),

(Apart from the complaint that your urine is yellow),

se ko de si any other complain min?

(Is it not there is any other complaint any?)

(Do you think there is no other complaint?)

Pat: Then, naa, ori maa ntun nfo mi, otutu.

(Then, again head is aching me, cold).

(Then, I also have headache and cold).

Doc: Un, nigba wo ni otutu yen ti bere?

(Yes; when did cold that has started?)

(Yes; since when has the cold started?)

Pat: Last week.

$\rightarrow$ Doc: Oju yin si pale. Ounje nko?

(Eyes your still pale. Food what about?)

(Your eyes are still pale. Do you eat?)

Pat: Mo njeun. 
(I am eating).

(I eat).

When Doc says "your eyes are still pale", he is mitigating the condition so as not to scare CL. Synonymous statements such as "You have problems with your blood. This is what accounts for your eye colour" may be frightening and damaging. Doc here simply appeals to his knowledge of CLs with a related condition and phase factor. The latter consideration is of more medical importance given the advancement of CL's pregnancy.

\subsubsection{Palliative goals:}

As said earlier, palliative goals are achieved through veiling, doublespeak and normalisation. Each of these is taken in turn.

Veils are employed to reduce pandemonium and fear. Abbronyms such as BID (Brought in Dead), DA (Dead already), DF (Dead foetus) and GO (dead) often signal to doctors and other medical professionals tension-relaxing cues which enable them to accommodate other emergency cases. The veils however place on them the responsibility to edge relations out of the communication by sticking with the concealment items while final medical actions continue on deceased CLs.

Treatment- related abbronyms such as "IV" (Intravenous injection) and "IM" (Intramuscular injection) serve as veils to reduce CL's, usually a child's, fear of injections. Either item has the effect to make children suppose being offered better treatment alternatives than injections. This however depends on the age of the child and the frequency at which the options have been explored. An interesting scenario that involved one of my daughters at age 2 is presented below:

Ex. 12:

Daughter: Yee::, yee::; abeye, injection, injection; yee::

(Yee:: yee::; injection, injection, injection; yee:-)

Father: $\quad$ It won't pain you; ko nii dun e

(It not pain you)

(It won't pain you)

Daughter: Yee::, yee::, pyease:::, yee::::

(Yee::, yee:: Please:::, yee:::

$\rightarrow$ Doctor: $\quad$ Look here. It is $I M, I M$, not injection. It will not pain you. Be a good girl. Daughter: (calms down)

The two-year old girl already knows the words "abere" (realised as "abeye", an agedependent choice) and "injection" as Yoruba and English synonyms and associates injecting with pain which she wishes to avoid. The father's promise of less pain does not improve the situation because it is a routine choice which has never fulfilled any sincerity condition (Searle 1969, 1979) each time it has been used. The alternative "IM" therefore works to calm down the little girl who seems to believe in the authority of the doctor (whom she seems to trust more than her father in this context). Also, because the term is new, the child perhaps sees it fulfilling a desirable sincerity effect.

Doublespeaks work to trivialise CL's condition. The only example for this is "Oh! God" for "gonorrhoea" whose use is often triggered by others factor. It has the 
potential to be misinterpreted as an expression of frustration on Doc's part, while shared assumptions cue medical interactants into the diagnostic truth in the common ground. One of the doctor respondents gave the following interaction example, which occurred when relations, including the sufferer's wife, brought a gonorrhoea case to his hospital:

Ex. 13:

$\rightarrow$ Doctor: $\quad$ (to an attendant nurse) Matron, this case, Oh! God, get me gloves (.)

Nurse: $\quad$ Alright sir

According to the doctor, the relations, especially the wife, who were all at the Intensive Care Unit of the hospital while the medical team attended to the sufferer, had thought the man was experiencing an end stage of some deadly disease, which confirmed to the doctor that the disease was an extramarital acquisition and that it had not been disclosed to the relations. Bonded to keep his client's secret, the doctor sandwiches the interjective, "Oh God!" in his directive to the nurse who already shares his beliefs. "Oh God!" in this context projects the doctor's frustration to the relations thereby confirming the relations' hypothesis of a deadly disease. But, it identifies a specific condition in the mental picture of the nurse. Her "alright sir" maps on the religious image to a medical condition, "gonorrhoea".

Normalisation, in the palliative goal, seeks to reduce the severity of CL's condition. From a doctor respondent's accounts, it is gathered that when the phrase "toilet infection" is used to refer to "yeast infection", they mean to provide CL with the impression that she is not experiencing a critical condition, which may not necessarily be so. Normalisation in the palliative programme differs from that in the preventive programme with respect to phase and others factors. Usually, even while the same phrasing is favoured in executing the two goals, normalisation in the palliative scheme applies when some symptoms are obvious which could not be denied but which have to be played down. Also, news announcement through normalisation is desired when a third party is present at the "disclosure space" (Glasser and Strauss 1965: 153), extended, in this paper, to all sites in the hospital environment where announcements are made.

\subsubsection{Culture-complaint goals:}

Culture-compliant goals are achieved only through veiling and euphemisation, and are associated in each case strictly with taboos. In other words, they are supported by the cultural doxas that obtain in South-western Nigerian society.

Some doctors prefer the simple abbronym "VE" for "vagina Examination" whether they are dealing with CL alone or with others in attendance. This tendency has been connected with the culture-based avoidance of direct naming of sexual organs in the Yoruba community (cf. Odebunmi 2010b). Like sexual organs, sexual intercourse is also generally indirectly referred to by doctors, which suits the cultural preference of most CLs. The interaction below shows how some taboo elements are interactively negotiated: 
Ex. 14:

Doc: $\quad$ How are you? $=$

Patient: $\quad$ It has come down again o:::! (sobs) $(0.3)$

Doc: ((looks through her case file)) No problem; you will be okay=

Patient: $\quad$ (still sobbing) (0.6)

Doc: $\quad$ Sorry Ma: dam, do you go to the farm every day? (0.4)

Patient: $\quad$ My husband does--, $<$ I sell provisions at home $>$ (.)

$\rightarrow$ Doc: $\quad$ How often does your husband sleep with you? (0.5)

$\rightarrow$ Patient: $\quad$ We used to do it often (0.3), but since the former doctor said we $\rightarrow$ should reduce $i t$, we have reduced it to two times a week (0.2).

Doc: $\quad$ Have you done abortion in the past? (0.8)

Patient: $\quad \operatorname{Hmm}(0.2),<\mathrm{I}$ had one when I was in the secondary school and three when I was living with my sister in Lagos>

Doc: $\quad$ Did you go to the hospital to do them? I mean the...

Doc in Ex.14 holds a conversation with CL who has just lost another pregnancy ("It has come down again o") which presupposes a sex-based encounter. Doc in a CR (cuebased request (Odebunmi 2010c)) asks: "how often does your husband sleep with you?" "Sleep with you" replaces "have sex with you" which is believed to be a blunt choice in a conversation with a married Yoruba woman. Therefore, though an obvious choice, "sleep with" is culturally expected in this context. Its concealment tone becomes reinforced in subsequent anaphoric ties. For example, CL, exploiting, in her response, the same cultural doxa observed by Doc, reaches for "do it", a contextual synonym for "sleep with", also avoiding direct reference "to have sex with". She tracks it subsequently with the anaphoric pronominal form "it": "... said we should reduce it, we have reduced it..."

\subsubsection{Confidential goals}

To achieve confidential goals, interactants appeal to veils with regard to pregnancyrelated conditions, hypertension/heart conditions, and sexual diseases. No interactional instances of concealment items related to the conditions were found in the data, but the respondent doctors gave a few examples as presented in the table below:

\begin{tabular}{|c|c|c|c|c|}
\hline $\mathbf{s} / \mathbf{n}$ & Strategy & $\begin{array}{l}\text { Concealment } \\
\text { Items }\end{array}$ & Linguistic Form & Full Form \\
\hline 1 & Veiling & $\begin{array}{l}\text { 1. BBA } \\
\text { 2. PT } \\
\text { 3. EDO } \\
\text { 4. D \& C } \\
\text { 5. STD } \\
\text { 6. CCF }\end{array}$ & $\begin{array}{l}\text { Simple Abbronym } \\
\text { Simple Abbronym } \\
\text { ComplexAbbronym } \\
\text { Simple Abbronym } \\
\text { Simple Abbronym } \\
\text { Simple Abbronym }\end{array}$ & $\begin{array}{l}\text { Born Before Arrival } \\
\text { Pregnancy Test } \\
\text { Expected Day of Delivery } \\
\text { Dilation and Curettage } \\
\text { Sexually Transmitted Disease } \\
\text { Congestive Cardiac Failure }\end{array}$ \\
\hline
\end{tabular}

Table 3: Confidential Goals

$1,2,4,5$, and 6 are simple abbronyms while 3 is a complex abbronym. Each of these, according to our respondent doctors, is employed with consideration for others factor. Hence, when used, doctors "restrict information and maintain confidentiality." 
It is interesting to note that the items are either socially or culturally motivated. "BBA", "CCF", "STD", and "D \& C" are socially constrained in that clients with the conditions do not want them announced because of possible complications (as in the case of "BBA"), potential danger of death (e.g. "CCF" and "D \& C") and problems of stigma (e.g. "STD" and "D \& C"). "EDO" and "PT" are culturally constrained. Culturally speaking, a typical Yoruba woman does not want her delivery date publicly pre-announced, as births are regarded as best when their news comes as a surprise. Hence, newly born babies are regarded as "visitors" in Yorubaland. This doxa also motivates the choice of "EDO" in place of the full form, "Expected Delivery Date". "PT"'s cultural association ties in with EDO's: until a pregnancy is self-noticeable, no one announces it. But "PT" also has a social slant, which comes into effect when unmarried women or heterosexual married women seek doctors' service. Both the doctor and client in this context collaboratively appeal to concealment considering others factor, which brings "PT", a largely private item into the communication.

\section{Conclusions}

By and large, it has been shown that concealment in South-western Nigerian hospitals takes place between doctors and clients in a two-phase mode: Referential and pragmatic. Utterances which at the referential level have descriptive forms assume subjective and divergent shades in the context of concealment. Nine concealment strategies (jargonisation, veiling, forecasting, mitigation, stalling, normalisation, dysphemisation, euphemisation and doublespeak) are employed to achieve four broad goals: preventive, palliative, culture-compliant and confidential. These findings have demonstrated a conflation of medical communication and pragmatic paradigms. Technical and plain communicative modes have been shown to undergo transformations largely within the ambits of doctors' goals

Concealment as practised in Nigerian hospitals is largely a safe-playing strategy. While the doctors do not claim to be ignorant of what medical ethics stipulates with respect to clarity on a client's condition, they still find practical social and cultural exigencies to present equivocal information to clients on certain conditions. The kind of experience in the interaction below, cited in Odebunmi (2003: 136), has been a basis for many doctors' decision on concealment:

Ex. 15.

Background: A patient had oesophageal achlazia from malignant metastasis. The surgeon (an American expatriate) addressed the medical team directly before the commencement of the operation on the patient thinking the man did not understand English:

$\rightarrow$ Doctor: $\quad$ This patient is having oesophageal, and he gonna live for just eighteen months (.)

Patient: $\quad$ Myself, me::, me:::, doctor:::: ((the patient fainted and dropped dead)).

That the client did not live up to the predicted 18 months was a direct consequence of lack of concealment at the critical stage of the client's illness. The American expatriate surgeon probably did not have an orientation in the kind of concealment tradition that is 
practised in many Southwestern Nigerian hospitals. This is coupled with his lack of knowledge about CL's socio-educational background which neither his physiognomy nor his deportment indexed. Invariably, even if the client would die ultimately, his life would have been prolonged a little further by a co-construction of the news with him in a more acceptable manner.

What is impressive about the practice of concealment in Nigerian hospitals which ties in well with standard medical practice is the temporary span of the strategies. About $90 \%$ of the doctor respondents and $60 \%$ of the client respondents expressed this opinion. Where concealment extends to longer temporal stretches, participants work on a common ground which supports concealment. The challenge that seems to attend the practice is the need to review many of the concealment tokens as they sneak to public knowledge and cease to be effective, which may, in the long run, make clients view language in the hospital critically when expressions used by doctors do not express clear propositions. This however could hardly obtain fully in South-western Nigerian hospitals where many clients take doctors on trust and are on several occasions led more by healing instincts than by critical language perspectives.

Concealment in Nigerian hospitals can be regarded as a practice that takes into account the socio-psychological security needs of clients and attend positively to clients' cultural expectations. Future research can explore in-depth concealment strategies employed in regard to killer diseases, especially HIV/AIDS, which takes the higher frequency in my rating in this study and which is a major global health menace, in the current face of some level of available effective medical intervention.

\section{References}

Buckman, Robert (2001) Communication skills in palliative care: A practical guide. Neurologic Clinics. 19 (4).http://www.ncbi.nlm.nih.gov/pubmed 11854110. Accessed May 20, 2010.

Capone, Alesandro (2005) Pragmemes: A study with reference to English and Italian. Journal of Pragmatics. 37: 1355-1371.

Chimombo, M., and R. Roseberry (1998) The Power of Discourse: An Introduction to Discourse Analysis. New Jersey: Lawrence Erlbaum Associates, Inc.

Fairclough, Norman (1989) Language and Power. London: Longman.

Frankel, R.M. (2001) Clinical care and conversational contingencies: The role of patients' self-diagnosis in medical encounters. Text 21: 83-111.

Glasser, Barney, and Anselm L. Strauss (1965) Awareness of Dying. Chicago, IL: Aldine.

Good, Mary-Jo, Byron Good, Cynthia Schaffer, and Stuart Lind (1990) American oncology and the discourse on hope. Culture, Medicine and Psychiatry 14: 59-79.

Heath, Collins (1992) Diagnosis and assessment in the medical consultation. In P. Drew and J. Heritage (eds.), Talk at Work: Interaction in Institutional Settings. Cambridge: Cambridge University Press, pp. 235-267.

Heritage, John, and T. Stivers (1999) Online commentary in acute medical visits: A method of shaping patient expectations. Social Science and Medicine 49: 1501-1517. 
Heritage, John, and Douglas Maynard (2006) (eds) Communication in Medical Care: Interaction Between Primary Care Physicians and Patients. Cambridge: Cambridge University Press.

Keskes, Istvan (2010) Situation-bound utterances as pragmatic acts. Journal of Pragmatics. 42.1: 28892897.

Leppanen, V. (1998) Structures of District Nurse-Patient Interaction. Lund, Sweden: Department of Sociology, Lund University.

Levinson, Stephen (1979) Activity types and language. Linguistics 17: 365-399.

Maynard, Douglas (1989) Notes on the delivery and reception of diagnostic news regarding mental disabilities. In D.T. Helm, W.T. Anderson, A.J. Meehan and A.W. Rawls (eds.), The Interactional Order: New Directions in the Study of Social Order. New York: Irvington, pp. 54-67.

Maynard, Douglas (1991) Interaction and asymmetry in clinical discourse. American Journal of Sociology 97: 448-495.

Maynard, Douglas (1996) On 'realisation' in everyday life: The forecasting of bad news as a social relation. American Sociological Review 16.1: 109-131.

Maynard, Douglas (2003) Bad News, Good News: Conversational Order in Everyday Talk and Clinical Settings. Chicago: University of Chicago Press.

Maynard, Douglas (2005) 'Does it mean I'm gonna die?': On meaning assessment in the delivery of diagnostic news. Social Science and Medicine.

Mey, Jacob (2001) Pragmatics: An Introduction. Oxford: Blackwell Publishers.

Odebunmi, Akin (2003) Pragmatic features of English usage in hospital interactions amongst medical practitioners and patients in South-western Nigeria. Unpublished Ph.D. thesis. Obafemi Awolowo University, Ile-Ife.

Odebunmi, Akin (2005) Politeness and face management in hospital conversational interactions in SouthWestern Nigeria. Ibadan: Journal of English Studies 2: 1-22.

Odebunmi, Akin (2006a) Meaning in English: An introduction. Ogbomoso: Critical Sphere.

Odebunmi, Akin (2006b) Locutions in medical discourse in South-Western Nigeria. Pragmatics 16.1: 2541.

Odebunmi, Akin (2007) Acts doctors and patients perform in medical encounters in Nigeria. In Moji Olateju, Rotimi Taiwo and Adeleke Fakoya (eds.), Towards Understanding Discourse Strategies. AgoIwoye: Olabisi Onabanjo University Press, pp.1-17.

Odebunmi, Akin (2008) Pragmatic strategies of diagnostic news delivery in Nigerian hospitals. Linguistik Online 36.4: 21- 37.

Odebunmi, Akin (2010a) Tracking ideology in political news. California Linguistic Notes XXXV (2).

Odebunmi, Akin (2010b) Ideology and body part metaphor in Nigerian English. Review of Cognitive Linguistics 8.2: 272-299.

Odebunmi, Akin (2010c) Code selection at first meetings: A pragmatic analysis of doctor- client conversations in Nigeria. Unpublished manuscript. 
Odebunmi, Akin, and M.A. Alo (2010) Beliefs in GSM text-messaging among academics in two Nigerian universities. In Rotimi Taiwo (ed.), Handbook of Research on Discourse Behaviour and Digital Communication: Language Structures and So-cial Interaction. New York: IGI Global, pp. 468-478.

Odebunmi, Akin, and Peter Auer (2011) Face, politeness and culture in doctor-client encounters in Nigeria. Unpublished manuscript.

Ong, LM, J.C. de Haes, A.M. Hoos, and F.B. Lammes (1995) Doctor-patient communication: A review of the literature. Public Medicine 40: 903-918.

Perakyla, Anssi (1998) Authority and accountability: The delivery of diagnosis in primary health care. Social Psychology Quarterly 61.4: 301-320.

Stivers, Tanya (1998) Prediagnostic commentary in veteranarian-client interaction. Research on Language and Social Interaction 31.2: 241-277.

The, Anne-Mei, Tony Hak, Gerard Koeter, and Gerrit van de Wal (2000) Collusion in doctor-patient communication about imminent death: An ethnographic study. Pubmed Journal.

http://www.ncbi.nlm.nih.gov/pmc/articles/PMC. Accessed May 20, 2010.

Tseng, Ming-Yu (2010) The pragmatic act of fishing for personal details: From choice to performance. Journal of Pragmatics 42: 1982-1996.

Williams, John (2009) Medical Ethics Manual. France: World Medical Association.

AKIN ODEBUNMI teaches discourse analysis, semantics and stylistics in the Department of English, University of Ibadan, Nigeria. His papers have appeared in Studia Anglica Posnaniensia, Pragmatics, Intercultural Pragmatics, Ibadan Journal of English Studies, Linguistik Online, California Linguistic Note, Marang, Ife Studies in English Language, Anglogermanica, Africa: Revista Do Centro De Estudos Africanos, Nordic Journal of African Studies, The International Journal of Language, Society and Culture and Review of Cognitive Linguistics. Between April 1, 2010 and March 31, 2011, he was an Alexander von Humboldt fellow at the Freiburg Institute for Advanced Studies, University of Freiburg, Germany.

Address: Senior Lecturer, Pragmatics, Discourse Analysis and Stylistics, Department of English, University of Ibadan, Ibadan, Nigeria. Tel.: 2348033786713. E-mail: akinodeb@yahoo.com 